\title{
Papers
}

\section{Appropriateness of use of medicines in elderly inpatients: qualitative study}

Anne Spinewine, Christian Swine, Soraya Dhillon, Bryony Dean Franklin, Paul M Tulkens, Léon Wilmotte, Vincent Lorant

\begin{abstract}
Objectives To explore the processes leading to inappropriate use of medicines for elderly patients admitted for acute care. Design Qualitative study with semistructured interviews with doctors, nurses, and pharmacists; focus groups with inpatients; and observation on the ward by clinical pharmacists for one month.

Setting Five acute wards for care of the elderly in Belgium. Participants 5 doctors, 4 nurses, and 3 pharmacists from five acute wards for the interviews; all professionals and patients on two acute wards for the observation and 17 patients (from the same two wards) for the focus groups.

Results Several factors contributed to inappropriate prescribing, counselling, and transfer of information on medicines to primary care. Firstly, review of treatment was driven by acute considerations, the transfer of information on medicines from primary to secondary care was limited, and prescribing was often not tailored to elderly patients. Secondly, some doctors had a passive attitude towards learning: they thought it would take too long to find the information they needed about medicines and lacked self directed learning. Finally, a paternalistic doctor-patient relationship and difficulties in sharing decisions about treatment between prescribers led to inappropriate use of medicines. Several factors, such as the input of geriatricians and good communication between members of the multidisciplinary geriatric team, led to better use of medicines.

Conclusions In this setting, improvements targeted at the abilities of individuals, better doctor-patient and doctor-doctor relationships, and systems for transferring information between care settings will increase the appropriate use of medicines in elderly people.
\end{abstract}

\section{Introduction}

Elderly patients are frequent users of health services and medicines. Research, however, has identified problems in the effective use of medicines in this population. ${ }^{12}$ Adverse drug reactions are implicated in $5-17 \%$ of hospital admissions. ${ }^{1}$ Elderly people are also less likely to receive treatments indicated by guidelines, ${ }^{3}$ such as those for patients admitted to hospital with myocardial infarction. ${ }^{4}$ In addition, discrepancies with medicines prescribed in the hospital occur after discharge. ${ }^{5}$ Although many quantitative studies have identified deficiencies, only limited work has been carried out on the reasons for these problems.
To optimise the provision of care in hospital and around discharge for elderly people, the national service framework for older people in the United Kingdom and similar strategies in other countries have encouraged the development of programmes of "care of the elderly." In this approach, multidisciplinary teams deliver medical, psychosocial, and rehabilitative care. The "single assessment process" was later developed to ensure that professional resources are used effectively. ${ }^{6}$ There is, however, limited qualitative or quantitative data on the appropriateness of use of medicines in elderly people admitted to acute care for the elderly wards. ${ }^{78}$

We explored the appropriateness of use of medicines for patients admitted to wards for care of the elderly from the perspectives of healthcare professionals and patients. We considered prescribing, counselling, and information given to the general practitioner at discharge.

\section{Methods}

\section{Study design}

We used individual semistructured interviews to explore the perspectives of relevant healthcare professionals. Observations on the ward were conducted to complement findings from interviews and to uncover behaviours that healthcare professionals may be unaware of. Finally, because pilot work showed some difficulties in stimulating discussion in individual interviews, we used focus groups to examine the views of elderly inpatients on issues relevant to them (changes in treatment and counselling).

\section{Sampling strategy}

The study was conducted on five acute wards for care of the elderly in five Belgian hospitals, purposively selected to include teaching and non-teaching, rural and urban settings. A multidisciplinary team of doctors, nurses, physiotherapists, social workers, and occupational therapists cared for patients. The purposive sample of five doctors and four nurses working on these five wards reflected variety in terms of position and experience (table). Three doctors were geriatricians, the others were house officers. We purposively selected three hospital pharmacists with relevant experience to complement the views of doctors and nurses.

Patients were recruited from two wards, one in an urban setting and the other rural. We purposively selected individuals able to share personal experience relating to changes in treatment and counselling. Such patients had to be stable and not

Details of the research process, interview schedules, and observation grid are on bmj.com 
Summary of participants' characteristics

\begin{tabular}{lcccc} 
Characteristics & $\begin{array}{c}\text { Doctors } \\
(\mathbf{n}=\mathbf{5})\end{array}$ & $\begin{array}{c}\text { Nurses } \\
(\mathbf{n}=\mathbf{4})\end{array}$ & $\begin{array}{c}\text { Pharmacists } \\
(\mathbf{n}=\mathbf{3})\end{array}$ & $\begin{array}{c}\text { Patients } \\
(\mathbf{n}=\mathbf{1 7 *})\end{array}$ \\
\hline No of women & 3 & 2 & 3 & 10 \\
\hline Age range (years) & $25-41$ & $31-44$ & $25-28$ & $73-92$ \\
\hline Teaching:non-teaching setting & $3: 2$ & $2: 2$ & $3: 0$ & $17: 0$ \\
\hline Experience in care of elderly (years) & $1-10$ & $1-30$ & NA & - \\
\hline Mean No of medicines on admission (range) & - & - & - & $7(3-12)$ \\
\hline Mean No of changes in treatment (range) & - & - & - & $7(3-12)$ \\
\hline
\end{tabular}

NA: not applicable because pharmacists in Belgium are not directly involved in care of patients on wards. These pharmacists were not involved in other parts of study.

${ }^{*}$ Four focus groups comprised three patients each. Five patients were interviewed individually.

confused; have no cognitive impairment; have had at least one modification in chronic medication; and had to manage their own medication at home. Two focus groups were conducted on each ward. Pilot work suggested that three to four patients per group worked best. The doctors identified eligible patients (table).

Observations occurred on the wards where focus groups took place over a one month period. Healthcare professionals, patients, and heads of departments gave informed consent after they had received oral and written information (see bmj.com).

\section{Instruments and data collection}

Interviews - AS conducted interviews using a guide piloted with two healthcare professionals external to the main study. Each interview lasted about an hour. Questions were open ended and covered perceived appropriateness of prescribing, counselling, and sharing of information relating to medicines, together with factors contributing to inappropriateness (see bmj.com).

Focus groups-An experienced independent researcher who was not involved in the rest of the study moderated each group. Key questions pertained to knowledge of treatment, satisfaction with changes in treatment, and information received (see bmj.com). Each discussion lasted about 45 minutes.

Observation-Two clinical pharmacists (AS and another pharmacist not involved in the rest of the study) observed all the main activities on two wards. Healthcare professionals were informed of their role, but did not know the extent of their observations. Observers described events relating to the use of medicines. When they identified inappropriate use (according to their clinical judgment) they informally discussed this with prescribers. Both observers took notes to remind them of key events and used an observation grid to write these notes up in more detail later (see bmj.com).

\section{Data processing and analysis}

All interviews (focus groups and the informal discussions with prescribers) were taped, transcribed, and entered into QSR NVivo (version 1.2) for support in coding and analysis. We used the principles of grounded theory ${ }^{9}$ to analyse the data with an inductive approach combining biomedical and sociological perspectives (see bmj.com).

\section{Results}

Most interviewees (especially those from teaching settings) admitted that prescribing was sometimes inappropriate and that counselling of patients was insufficient. Observations and data from focus groups corroborated these findings. In addition, one geriatrician and all pharmacists thought that the information shared with the general practitioner on discharge was insufficient. Observers confirmed that it was often limited to a list of medications.
Three main categories underlying inappropriate use of medicines emerged (box). All three contributed to inappropriate prescribing. The third category (paternalistic decision making) contributed to most instances of inappropriate counselling and ineffective transfer of information.

\section{Reliance on general acute care and short term treatment}

Most participants thought that they devoted considerable time to acute problems and that prescribing for chronic diseases was overlooked. Observations confirmed this. Reasons included insufficient incentives to review chronic problems in an acute care setting.

I think that doctors pay a lot of attention to the acute problem, but they don't give enough consideration to other medicines that patients are on. For example, a patient had been admitted for syncope secondary to atrial fibrillation. They started to give digoxin to control the fibrillation. But at the same time, pain care, for example, was inadequate: the patient was on paracetamol and amitriptyline at home, and these were not re-prescribed in the hospital (observer 2).

Undermedication, it's important, but we don't consider that issue enough. It's clear that we don't treat hypertension enough, for example. And that's maybe more difficult in the hospital because we are in acute care, and so we first see the problem that brings the patient into hospital (doctor 5, geriatrician).

In addition, medicines (mainly for chronic conditions) were sometimes not appropriately reviewed because there was no written information on indication and follow-up or because this information was not readily available. This was identified through observations and subsequently validated by most prescribers.

Patient on fentanyl patch in nursing home, continued during admission. No indication in the medical notes; no report of pain; patient not communicative. When asked by the geriatrician, the house officer said she didn't know the indication. No change was made to the treatment afterwards, and there was no comment in the discharge letter. On being asked, the house officer later told me that treatment hadn't been reviewed because the indication wasn't known (observer $1)$.

Finally, several interviewees said that prescribing was often not tailored to elderly patients. For example, the dose was not adjusted to renal status, medicines for which risks outweighed benefits were used, or the formulation was inappropriate. This mainly happened with junior doctors and external consultants.

\section{Categories underlying inappropriate use of medicines}

Reliance on general acute care and short term treatment

- Review of treatment driven by acute considerations; other considerations overlooked

- Limited transfer of information on medicines from primary to secondary care

- "One size fits all": prescribing behaviour not tailored to the older patient

Passive attitude towards learning

- Anticipated inefficiency in searching for medicines information

- Reliance on being taught (teacher centred) rather than self directed learning

Paternalistic decision making

- Patients thought to be conservative

- Patients declared as unable to comprehend

- Ageism

- Difficulty in sharing decisions about treatment with other prescribers 
When house officers come on our ward, they haven't necessarily been trained in geriatrics. So they arrive here, and then they start with $10 \mathrm{mg}$ of morphine every four hours. That's too much (doctor 2 , geriatrician).

The formulations prescribed aren't always suited to the ability of an elderly person to swallow the medicine or to receive an injection, for example... The doctor doesn't necessarily think about it [the formulation prescribed]. I would even say that, except for Dr X [geriatrician], who is used to doing so, the house officers don't have this instinct (nurse 2).

\section{Passive attitude towards achieving learning outcomes}

In some cases doctors acknowledged that questions on medication (especially relating to interactions and side effects), when not answered by a colleague, remained unanswered because of anticipated inefficiency in accessing information on medicines. Observers also reported this

[House officer talking about drug interactions with warfarin, leading to increased international normalised ratio-that is, overanticoagulation] I still don't really know them well. And to always go and look in the compendium [a reference book with scientific information on licensed medicines] is a bit difficult in terms of time. I think that's the main reason why we don't check (doctor 3 , house officer).

In addition, several doctors thought that the learning process of house officers was passive and teacher centred rather than active and self directed. Doctors gave two explanations for this: low perceived interest and motivation on medication matters during undergraduate studies, and lack of time for active learning during training. As a consequence, junior doctors relied heavily on superiors' comments. There was a risk of passive application of "recipes" (also with medicines requiring special considerations on prescribing, see below). Observations corroborated this finding.

When we were studying, it was not really compulsory for us to take a serious interest in the literature. And I used to always say to myself "when I'm a house officer, I will read it up." Well in everyday practice we rely very much on our superiors' comments. I hardly ever go and look up what to do myself, what is the right thing to do ... Also because that takes longer to do (doctor 1 , house officer).

Observation in relation to the care of an 80 year old man complaining of insomnia] The geriatrician suggested that the house officer prescribe a preparation of chloral hydrate. The house officer prescribed it, using the formula available in the office. She later told me that she didn't know this drug at all, but that apparently the geriatrician was used to prescribing it. Contraindications and drug interactions had not been considered (observer 1 )

\section{Paternalistic decision making}

Most participants agreed with the identified factors relative to paternalism, but several doctors said that the first three did not always occur (see box). Most doctors and nurses thought that changes in treatment were often difficult to implement because patients were attached to their usual medicines.

For example, for an antidepressant that had been given for a minor depression and that the patient is on for life, that nobody tried to stop a year or so later, well they [patients] are attached to it, it's difficult to go against that (doctor 2, geriatrician).

Conservatism also applied to counselling. One nurse described the unwillingness to inform patients of side effects.

I've noticed before, too, that they [patients] weren't told about known side effects because it was thought that they would be afraid of taking the medicine or that they would start feeling those side effects (nurse 4).

Several interviewees thought that the problem underlying conservatism was insufficient decision sharing.
I think that if somebody explains to the patient why he or she is given this medicine, the patient could understand. Here patients mightn't understand, quite simply because they [doctors or nurses] don't know how to explain things to them (pharmacist 3).

I think that too often, they don't ask what the patient thinks. For example, when a patient comes into hospital, they replace his laxative, $\mathrm{X}$, by another laxative, Y. It mightn't seem that important, but for the elderly person it is. Even just from a psychological point of view, I would say (nurse 3).

Shortage of time and an assumption of inability to comprehend were other reasons for insufficient counselling.

Some of the patients wouldn't take it in [information on the indication of each medicine], because, well, two thirds of our patients have cognitive impairment, after all (doctor 4 , geriatrician).

The attitude of most patients regarding treatment decisions reinforced a paternalistic model.

The doctors tell me, "We'll stop this one and give you something else that will work better." Well, for me that's fine. I have boundless confidence in them.

With regard to counselling, however, about half of patients expressed dissatisfaction with not being informed about changes.

I'm completely lost ... My medicines were replaced by different ones, but I don't know who decided that ... and I don't know what they are. . .. I would like to know what I'm taking and what I am being treated for. I like to know the "why" (patient).

The group discussions helped patients clarify their thoughts but they did not change their views.

One doctor cited ageism to explain underuse of medicines.

I think that some illnesses don't get enough treatment ... probably in part due to what is called ageism. You say to yourself, What good will it do? Why add more medication? Is it worth optimising treatment? (doctor 5 , geriatrician).

Finally, most doctors, two nurses, and both observers identified difficulties in sharing treatment decisions between prescribers. This was for two reasons. Firstly, doctors were reluctant to interfere with treatment delivered by a colleague.

A patient had heart failure, NYHA [New York Heart Association] stage II. I asked the house officer why she was not getting an ACE inhibitor. He answered: "In fact ACE inhibitors are a first choice in heart failure and this patient is not getting them, but she is under the care of a cardiologist, so I'm not going to change the treatment" (observer 2).

Secondly, two doctors acknowledged that information transferred to general practitioners could be limited by fear of offending them with comments on inappropriate prescribing.

Just yesterday I saw a patient whose general practitioner had prescribed metoclopramide, although she has very severe Parkinson's disease. Well I can't really write in the letter that ... We're always afraid of offending (doctor 2, geriatrician).

\section{Processes leading to appropriate medicines use}

Besides the identification of processes underlying inappropriate use of medicines, participants and observers described several factors that acted as a stimulus for treatment review.

A first stimulus to review treatment was the perceived excessive number of medicines taken by the patient. Patients were often thought to take excessive numbers of medicines. This was mentioned by four doctors, two pharmacists, and one observer.

We often say among ourselves, "More than five, that's too many." When we exceed five medicines then one has to think, Is that really justified? (doctor 4, geriatrician). 
The identification of drug related problems by other members of the multidisciplinary team and subsequent communication to the prescriber also helped to optimise treatment.

For example, a patient that the physiotherapist gets to stand up, he could walk with his Rolator [walking frame with wheels], but then they had to add melperone [a neuroleptic with cardiovascular and central nervous system side effects], and he can't stand up any more. The physiotherapist will talk to the doctor and might ask if it could be linked to a change in his medication (nurse 2).

When nurses find our tablets too big, for example ... they ask me to find something else because it will never go down (doctor 3, house officer).

One doctor, one nurse, and one observer reported that a move from a curative to a palliative approach was an opportunity to reflect on the objectives of therapy and change treatment.

Sometimes people have taken 10 medicines while they were in curative care, and gradually they move on to palliative care. Then we must reconsider all the prescriptions, drug by drug, saying: OK, what's the goal? To improve your comfort? Well, this medicine will make you feel more comfortable; we can stop this other one (doctor 5 , geriatrician)

Finally, several doctors and pharmacists perceived that the input of geriatricians was valuable to counteract the "one size fits all" approach. Observed events confirmed this.

When I see a patient who is on prazepam, for example [a benzodiazepine to be avoided in elderly people because of its long half life], well, I often ask for a review of the prescription, and to see if it wouldn't be more appropriate to select a drug with a shorter half life, for example (doctor 2, geriatrician).

\section{Discussion}

Reliance on general acute care and short term treatment, passive attitudes towards learning, and paternalism can all lead to inappropriate use of medicines in elderly people. We analysed data from the perspectives of professionals and patients (theory and data triangulation), using a combination of methods (methodological triangulation).$^{10}$

\section{Reliance on general acute care and short term treatment} Considerations relating to the treatment of chronic conditions relating to treatment are sometimes overlooked, one reason being the nature of an acute care setting. Though this is a worldwide issue ${ }^{11}$ this is the first report of occurrence on wards for care of the elderly. Such considerations did not seem to apply to events of overt polymedication or of palliative care. Another reason was the limited transfer of information on treatment between primary and secondary care. This highlights the importance of improving continuity of care. ${ }^{12}$ It contrasts with the reported benefit of oral communication in the multidisciplinary team for care of the elderly. Another issue, which is also worldwide, was the lack of adequate training of doctors in prescribing for geriatric patients. ${ }^{13}$

\section{Passive attitude towards achieving learning outcomes}

Previous work found that "lack of knowledge" and "lack of time" contribute to suboptimal prescribing. ${ }^{15}$ This was often cited by participants in our study. We have also identified new explanatory factors behind these rather descriptive terms: doctors anticipated inefficiency in accessing information about medicines and junior doctors had a passive attitude towards learning. This is worrying because most prescribing errors are made by junior medical staff. ${ }^{16}$

\section{Paternalistic decision making}

Paternalism may lead to inappropriate use of medicines. Provision of information should be tailored to individual needs. ${ }^{17}$
This reinforces the importance of patient empowerment. ${ }^{18}$ Similarly to findings from other settings, some patients wanted information but the doctor did not realise this or thought the patients did not need to know or would not understand. ${ }^{19}$

Ageism can be viewed as a form of paternalism. Together with "acute care" reasons, it led to events of underuse. However, there may be other reasons for undertreatment. Common explanatory themes in the literature are conceptualisation of illness and ageing, socioeconomic factors, allocation of resources, and provision of information. ${ }^{20}$

Finally, the findings show that decision making is further complicated because it often involves more than one prescriber. ${ }^{18}$ The reluctance to interfere with treatment prescribed by a colleague was, interestingly, not reported in previous qualitative studies on appropriateness of medicines use. A similar issue, however, was raised in a quantitative study. ${ }^{21}$

\section{Weaknesses}

Generalisability is an issue because our study involved a limited number of respondents in a limited number of hospitals. A comparison of the results with previous qualitative studies (cumulative validation) showed that some factors had often been identified in other settings, while others had not. For the latter factors we do not know whether they are specific to the local or national setting from which the sample was drawn.

We cannot exclude the occurrence of a researcherrespondent interaction (Hawthorne effect) during interviews and observations. This was minimised by presenting research objectives in a constructive way and by using a disguised observation technique. Most interviewees were not reluctant to talk about problems, and the presence of observers was well accepted. It is possible, however, that healthcare professionals paid more attention to medicines when observers were present. ${ }^{22}$

\section{Conclusions and implications}

Elderly people often have several chronic conditions and need several medicines, are often admitted to hospital, and need regular review of treatment. ${ }^{1}$ Optimising use of medicines throughout a hospital stay is therefore highly relevant to this population.

We identified several factors contributing to inappropriate medicines use. Some have been described in previous studies (reliance on general acute care, paternalistic doctor-patient relationship), while others are rather new (factors relating to the learning attitude and to relationships between prescribers). Strategies for improvement should include approaches such as developing incentives for considerations relative to the treatment of chronic conditions and for active learning in geriatrics by junior doctors ${ }^{23}{ }^{24}$; developing systems for reliable transfer of information; and increasing involvement of patients and encouraging constructive communication between prescribers. The input of geriatricians, as well as active multidisciplinary communication, should be encouraged. It might be interesting to study to what extent collaboration with clinical pharmacists could help overcome some of the barriers described.

We thank the participants who gave up their time to be interviewed. We also thank Michel Lambert and Felicithy Smith for helpful comments about the study design; Dominique Paulus and Jean-Marc Feron for conducting focus groups; Stéphanie Arman for conducting the second set of observations; Françoise Van Bambeke for the analysis of reliability; Jean-Marc Feron for revising quotes; and Martin McGarry for help with translation of quotes from French

Contributors: AS and CS had the idea for the study. AS, VL, CS, and SD designed the study. CS advised on recruitment. AS recruited and interviewed healthcare professionals. AS and VL developed the coding 


\section{What is already known on this topic}

Quantitative studies have identified problems in the use of medicines for elderly patients, including inappropriate prescribing, counselling of patients, and transfer of information between primary and secondary care

There is limited qualitative data on the processes underlying inappropriate use of medicines in older inpatients

\section{What this study adds}

Reliance on general acute care and short term treatment, passive attitudes towards achieving learning outcomes, and paternalistic decision making contribute to inappropriate use of medicines in elderly patients

The input of geriatricians and communication between members of a multidisciplinary geriatric team contributed to a better use of medicines

framework. All authors helped to analyse and interpret the data. AS and VI wrote the paper, and all authors revised it. AS is guarantor.

Funding: AS is a research fellow of the Belgian Fonds National de la Recherche Scientifique (FNRS).

Competing interests: None declared.

Ethical approval: The present study was part of a global project approved by the local ethical committee. The overall project was approved by the Belgian National Foundation for Scientific Research and by the $\mathrm{PhD}$ university research committee.

1 National Service Framework for Older People. Medicines and older people-implementing medicines-related aspects of the NSF for older people. London: Department of Health, 2001. the NSF for older people. London: Department of Health, 2001. Hanlon JT, Shimp LA, Semla TP. Recent advances in geriatrics: drug-related problems
in the elderly. Ann Pharmacother 2000;34:360-5.

3 Rochon PA, Gurwitz JH. Prescribing for seniors: neither too much nor too little.JAMA 1999;282:113-5

4 Rathore SS, Mehta RH, Wang Y, Radford MJ, Krumholz HM. Effects of age on the quality of care provided to older patients with acute myocardial infarction. Am J Med 2003; 114:307-15.

5 Burns JM, Sneddon I, Lovell M, McLean A, Martin BJ. Elderly patients and their medication: a post-discharge follow-up study. Age Ageing 1992;21:178-81.

6 Department of Health. Single assessment process. www.dh.gov.uk/PolicyAndGuidance/ HealthAndSocialCareTopics/SocialCare/SingleAssessmentProcess/fs/en (accessed 26 April 2005).

7 Owens NJ, Sherburne NJ, Silliman RA, Fretwell MD. The senior care study. The optimal Owens NJ, Sherburne NJ, Silliman RA, Fretwell MD. The senior care study. The op
use of medications in acutely ill older patients. J Am Geriatr Soc 1990;38:1082-7.

8 Schmader KE, Hanlon JT, Pieper CF, Sloane R, Ruby CM, Twersky J, et al. Effects of geriatric evaluation and management on adverse drug reactions and suboptimal prescribing in the frail elderly. Am J Med 2004;116:394-401.
9 Strauss A, Corbin J. Basics of qualitative research: grounded theory, procedures and techniques. London: Sage, 1990

10 Mays N, Pope C. Qualitative research in health care. Assessing quality in qualitative research. BMJ 2000;320:50-2

11 Cook DJ, Montori VM, McMullin JP, Finfer SR, Rocker GM. Improving patients' safety locally: changing clinician behaviour. Lancet 2004;363:1224-30.

$12 \mathrm{Al}$ Rashed SA, Wright DJ, Roebuck N, Sunter W, Chrystyn H. The value of inpatient pharmaceutical counselling to elderly patients prior to discharge. Br J Clin Pharmacol 2002;54:657-64.

13 Larson EB. General internal medicine at the crossroads of prosperity and despair: caring for patients with chronic diseases in an ageing society. Ann Intern Med 2001;134:997-1000.

14 Tomlin Z, Humphrey C, Rogers S. General practitioners' perceptions of effective health care. BMJ 1999;318:1532-5.

15 Dean B, Schachter M, Vincent C, Barber N. Causes of prescribing errors in hospital inpatients: a prospective study. Lancet 2002;359:1373-8.

16 Dean B, Schachter M, Vincent C, Barber N. Prescribing errors in hospital inpatients: their incidence and clinical significance. Qual Saf Health Care 2002;11:340-4.

17 Dickinson D, Raynor DK. What information do patients need about medicines? Ask the patients-they may want to know more than you think. BMJ 2003;327:861.

patients-they may want to know more than you think. BMJ 2003;327:861.
18 Gafni A, Charles C, Whelan T. The physician-patient encounter: the physician as a perfect agent for the patient versus the informed treatment decision-making model. Soc Sci Med 1998;47:347-54

19 Britten N, Stevenson FA, Barry CA, Barber N, Bradley CP. Misunderstandings in prescribing decisions in general practice: qualitative study. BMJ 2000;320:484-8.

20 Mold F, McKevitt C, Wolfe C. A review and commentary of the social factors which influence stroke care: issues of inequality in qualitative literature. Health Soc Care Community 2003;11:405-14.

21 Kroenke K, Pinholt EM. Reducing polypharmacy in the elderly. A controlled trial of physician feedback. JAm Geriatr Soc 1990;38:31-6.

22 Mangione-Smith R, Elliott MN, McDonald L, McGlynn EA. An observational study of antibiotic prescribing behavior and the Hawthorne effect. Health Serv Res 2002;37:1603-23.

23 Thomas DC, Leipzig RM, Smith LG, Dunn K, Sullivan G, Callahan E. Improving geriatrics training in internal medicine residency programs: best practices and sustainable solutions. Ann Intern Med 2003;139:628-34.

24 Rolfe IE, Sanson-Fisher RW. Translating learning principles into practice: a new strategy for learning clinical skills. Med Educ 2002;36:345-52.

(Accepted 28 June 2005)

doi 10.1136/bmj.38551.410012.06

Centre for Clinical Pharmacy, School of Pharmacy, Université catholique de Louvain, 1200 Brussels, Belgium

Anne Spinewine research fellow

Paul M Tulkens professor of pharmacology and pharmacotherapy

Department of Geriatric Medicine, Mont-Godinne University Hospital, 5530 Yvoir, Belgium

Christian Swine professor in geriatrics and gerontology

School of Pharmacy, University of Hertfordshire, Hatfield, Herts AL10 9AB Soraya Dhillon head of pharmacy

Academic Pharmacy Unit, Hammersmith Hospitals NHS Trust, London W12 0HS Bryony Dean Franklin director

Cliniques Universitaires Saint-Luc, Université catholique de Louvain, Brussels Léon Wilmotte chief pharmacist

School of Public Health, Université catholique de Louvain, Brussels

Vincent Lorant sociologist

Correspondence to: A Spinewine anne.spinewine@facm.ucl.ac.be 